\title{
Comparison between the robo-horse and real horse movements for hippotherapy
}

\author{
Ji H. Park ${ }^{\mathrm{a}}$, Timothy Shurtleff ${ }^{\mathrm{b}}$, Jack Engsberg ${ }^{\mathrm{b}}$, Sandy Rafferty ${ }^{\mathrm{c}}$, Joshua Y. You ${ }^{\mathrm{d}}$, Isaac Y. \\ You $^{\mathrm{e}}$ and Sung H. You ${ }^{\mathrm{a}, *}$ \\ ${ }^{a}$ Movement Healing Laboratory, Department of Physical Therapy, Yonsei University, 1 Yonseidae-gil, \\ Wonju 220-701, Republic of South Korea \\ ${ }^{b}$ Human Performance Laboratory, Program in Occupational Therapy, Washington University in \\ Saint. Louis, School of Medicine, 4444 Forest Park Ave. Box 8505, St. Louis, MO USA \\ ${ }^{c}$ Therapeutic Horsemanship, 332 Stable Lane, Wentzville, MO USA \\ ${ }^{d}$ Wydown Middle School, 6500 Wydown, Clayton, MO USA \\ ${ }^{e}$ Clayton High School, \#1 Mark Twain Circle, Clayton, MO USA
}

\begin{abstract}
While the novel robotic hippotherapy system has gradually gained clinical application for therapeutic intervention on postural and locomotor control in individuals with neurological or musculoskeletal impairments, the system's validity and reliability for the robotic hippotherapy system has not been well established. The objective of the current study was to investigate the validity and test-retest reliability of the robotic hippotherapy system by comparing with real horse movements. The 3-axis accelerometer sensors attached on the robotic and real horse saddles were used to collect 3-dimensional acceleration data at a preferred walking velocity. Linear regression analysis showed an excellent correlation in the time-topeak acceleration (TPA) $\left(R^{2}=0.997\right)$, but little correlation in $\mathrm{X}$-axis acceleration between the real and robotic horses $\left(R^{2}=0.177\right)$, thus confirming consistent time control and a certain degree of variability between the robotic and real horse movements. The mean resultant accelerations for a real horse and robotic horse were $3.22 \mathrm{~m} / \mathrm{s}^{2}$ and $0.67 \mathrm{~m} / \mathrm{s}^{2}$, respectively, accounting for almost five times greater acceleration in the real horse than the robotic horse.
\end{abstract}

Keywords: Robotic hippotherapy, acceleration

\section{Introduction}

Hippotherapy has gained clinical acceptance for improving postural stability, reaching and locomotor performance, and is a promising alternative rehabilitation technique for adults and children with neurological impairments [1-6]. Clinical hippotherapy studies have demonstrated significant improvements in static balance and dynamic reaching function after 12 weeks of hippotherapy in children with cerebral palsy (CP) $[7,8]$. Moreover, hippotherapy is beneficial for postural stability and locomotion in adults with hemiparetic stroke $[9,10]$. Hence, robotic hippotherapy has recently been

\footnotetext{
*Corresponding author: Sung H. You, Movement Healing Laboratory, Department of Physical Therapy, Yonsei University, 1 Yonseidae-gil, Wonju 220-701, Republic of South Korea. Tel.: +82 33760 2476; Fax: +82 33760 2496; E-mail: neurorehab@yonsei.ac.kr.
}

0959-2989/14/\$27.50 @ 2014 - IOS Press and the authors. 
integrated into conventional neurorehabilitation as an alternative choice for hippotherapy in a clinical environment or in centers where real horses are not readily accessible or affordable. This increase in utilization of robotic horses may have partly resulted from inherently limited accessibility, weatherdependence, and cost in the real horse hippotherapy programs [11]. The development process of horseback riding simulators have been discussed in great details from kinematic motion analysis to potential application in the literature. Both studies provide useful information about the development process of a legged multiple (6-) degrees of freedom horseback riding simulator for a safe and correct training of a novice rider [12,13]. Nevertheless, there is a dearth of studies about the robotic horse system which specialized in diagnosis and neurorehabilitation of children with CP.

Albeit devoid of ecologically natural interactions with a real horse, robotic hippotherapy has superior advantages including performing the therapy on a regular basis, no apparent spatiotemporal constraints, and weather independence [14]. The present robotic hippotherapy system (FORTIS-102, Daewon Fortis, HaNam, Kyungi, South Korea) is designed to facilitate stretching, rhythmic trunk rotations, core stabilization, strength, endurance, and cardiopulmonary function via the sensorimotor system (vestibular, proprioceptive) in children with moderate to severe CP. However, the robotic hippotherapy system's reliability and validity have yet to be determined for clinical application. Therefore, the present study compares movement of the robotic horse and several real horses by comparing acceleration kinematics between robotic and real-horses' walking movement patterns.

\section{Materials and methods}

\subsection{Materials}

The robotic hippotherapy system's (FORTIS-102, Daewon Fortis, HaNam, Kyungi, South Korea) preprogrammed anthropometric and spatiotemporal data are presented in Table 1. The system is primarily composed of three actuators (sliding assembly, elevating assembly, driving assembly), three high torque standard servo motors with dual ball bearings, and a saddle support, suspension frame and belt to provide safe and precise lumbopelvic motor control for locomotion. As illustrated in Figure 1, the system has special features: 2 degrees of freedom, adjustable speed and pattern (e.g., walking, trotting, and galloping) control, and a suspension chest belt and harness for safe and upright postural control.

This system is engineered to simulate real horse movements including a walk $(6 \mathrm{~km} / \mathrm{h})$ and trot $(15$ $\mathrm{km} / \mathrm{h}$ ). The exercise modes include 100 different 2-dimensional movement patterns and cycles. Some of the exercise modes approximate sagittal plane movements of a horse at various gaits and cadences, but still lack lateral movement. Thus the robotic horse provides rhythmic movement in only two of the three dimensions provided by real horses. The robotic horse also does not move through space, so it also does not provide the constantly changing visual flow and additional challenge to postural control that is afforded by a real horse when it changes direction while moving forward.

To determine which rhythmic movement patterns would be used for comparative analysis, three experienced horse riders rode the robotic horse and provided their subjective impressions of the movement patterns and speed settings which most closely approximated real horse movement at the walk. Based upon their impressions one pattern was selected for comparison to the walk of real horses for this investigation. 
Table 1

Anthropometric characteristics of the robotic hippotherapy system

\begin{tabular}{ll}
\hline Parameters & \\
\hline & Robotic hippotherapy system \\
Anthropometric data & \\
Width & $0.58 \mathrm{~m}$ \\
Length & $1.90 \mathrm{~m}$ \\
Height of the saddle & $1.30 \mathrm{~m}$ \\
Weight & $315 \mathrm{~kg}$ \\
Spatiotemporal data & \\
velocity & Adjustable in 100 steps \\
\hline
\end{tabular}

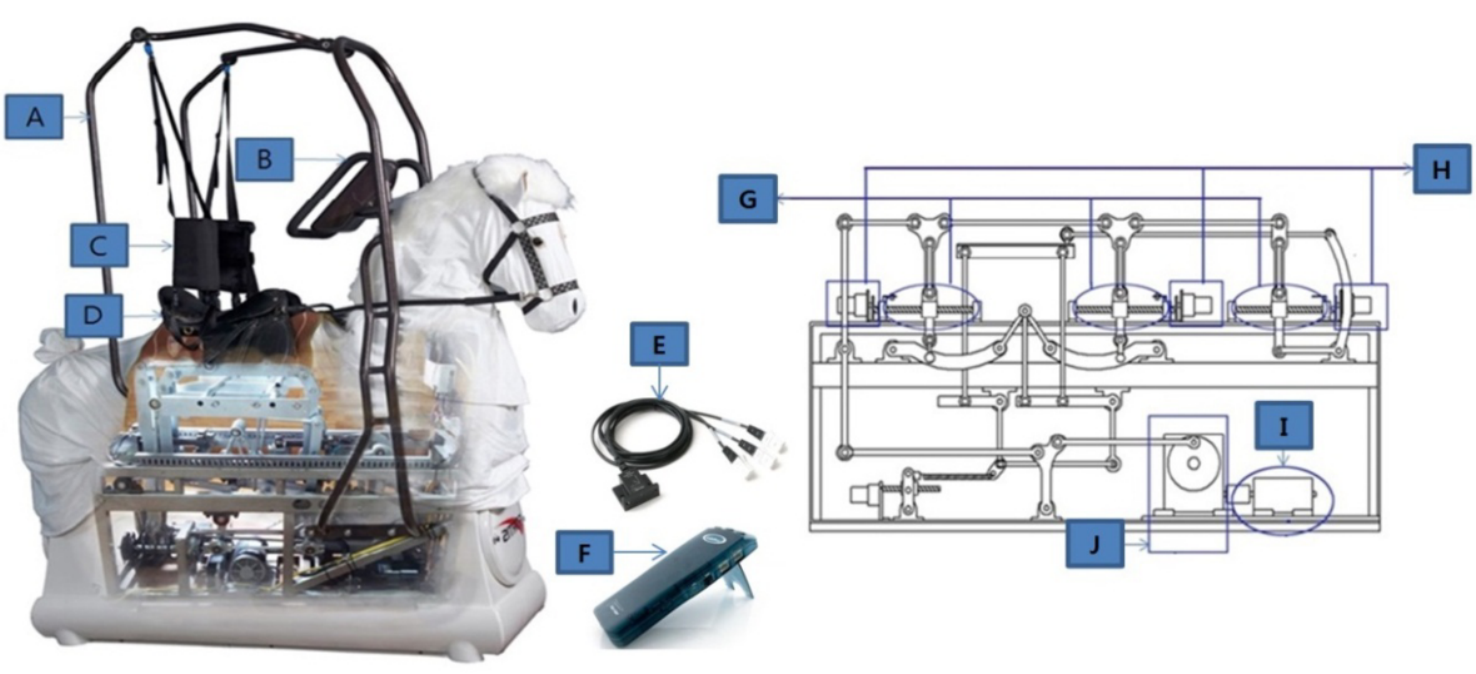

Fig. 1. Robotic hippotherapy system and experimental setup. A: suspension frame, B: controller, C: harness, D: saddle, E: 3axis accelerometer sensors, F: LabPro interface, G: ball screw, H: servomotor, I: pulley, J: main motor.

\subsubsection{Instrumentation}

The two 3 -axis accelerometer comprising three -5 to $+5 \mathrm{~g}$ accelerometers (Range: $\pm 49 \mathrm{~m} / \mathrm{s}^{2}( \pm 5 \mathrm{~g})$; Frequency Response: $0-100 \mathrm{~Hz}$; Resolution: $0.037 \mathrm{~m} / \mathrm{s}^{2}$ ) were used collect acceleration data (X-axis, Anterioposterior; Y-axis, Mediolateral; Z-axis, Vertical) because it is portable, accurate, and has less motion artifact associated with horse movement unlike the conventional motion analysis system such as Vicon which is stationary and not readily accessible for the horses or in most stables. Interface (LabPro, Vernier Software \& Technology, Oregon, USA) was used to connect with the accelerometer sensors and software (Logger Pro3, Vernier Software \& Technology, Oregon, USA) was used to compute the 3-axis acceleration and graphically display it on the monitor.

\subsection{Experimental procedure}

\subsubsection{Data acquisition}

The real horse acceleration and walking speeds were determined using the 3-axis accelerometer sensors mounted on a saddle. The acceleration data were collected from seven medium size real horses at their preferred gait speed in a barn (approximately $100 \times 70 \mathrm{~m}$ ). The digital motion sensors were 
connected to LabPro and the digital ports (DIG/SONIC), located on the same side as the serial and USB computer connections, were connected the laptop computer. Once average horse movement and speed were established, the estimated walking speed was programmed into the robotic horse system (i.e., movement pattern \#42, speed mode: 52) to resemble that of a real horse. Acceleration data were acquired at a sampling rate of $50 \mathrm{~Hz}$ over a 30 second period for both real and robotic horses.

\subsubsection{Data analysis}

The collected data were exported and stored as CSV file for further statistical analysis. The Logger Pro software was used to compute $\mathrm{X}, \mathrm{Y}$, and Z-acceleration and resultant acceleration, which was expressed: $\mathrm{a}=\frac{d V}{d t}=\frac{d v}{d t} u_{t}+v(t) \frac{d u_{t}}{d t}=\frac{d v}{d t} u_{t}+\frac{v^{2}}{R} u_{n}$ where $v(t)$ represents the changing speed, $u_{t}$ represents the changing direction, $u_{n}$ is the unit (outward) normal vector, and $\mathbf{R}$ is its instantaneous radius. Among the total acceleration movement data aquired for 30 seconds (1500 data points), the most consistent and representative $\mathrm{X}$-axis acceleration data points obtained for 5 seconds of movement $(\mathrm{N}=251)$ was used for correlation analysis across all of the horses and the robohorse. Then, the correlation matrix was computed as two variables (each real horse vs. robotic horse; or between real horses) were compared.

\subsection{Statistical analysis}

Quantitative kinematic analysis has commonly utilized acceleration and time-to-peak acceleration (TPA) to evaluate movement patterns in human and animal models because it provides accurate information about spatiotemporal parameters [15]. Linear regression analysis was computed to determine the validity and test-retest reliability of the robotic horse and real horses's acceleration data and resultant acceleration data collected. Additionally, TPA analysis was compared to determine potential similarities in time factor between the real and robotic horses. Significance was set at $p<0.05$. We used SPSS version 20.0 for Windows (SPSS, Chicago, IL, USA).

\section{Results}

\subsection{Comparison of the robotic horse to real horses}

To determine the validity of the robotic horse, first the TPA analysis with resultant acceleration data was compared between the seven real and robotic horses. Mean X-axis acceleration data was also compared. Linear regression analysis showed an excellent correlation in the TPA $\left(R^{2}=0.997\right)$ (Figure $2)$, but little correlation in $\mathrm{X}$-axis acceleration between the real and robotic horses $\left(R^{2}=0.177\right)$, thus confirming consistent time control and a certain degree of variability between the robotic and real horse movements.

Further movement analysis showed that the mean resultant accelerations for a real horse and robotic horse were $3.22 \mathrm{~m} / \mathrm{s}^{2}$ and $0.67 \mathrm{~m} / \mathrm{s}^{2}$, respectively, accounting for approximately 4.8 times greater acceleration in the real horse than in the robotic horse. A high degree of variability was observed among the seven real horses in Table 2 . 


\subsection{Test-retest reliability of the robotic and real horse movements}

To determine the test-retest reliability of the robotic and real horse movements, we consistently computed mean $\mathrm{X}$-axis acceleration data obtained from both the robotic horse and seven real horses.

A separate linear regression analysis showed good to excellent test-retest reliability for both robotic $\left(R^{2}=0.976\right)$ and real horse $\left(R^{2}=0.865\right)$ movements, indicating that the robotic and real horse acceleration movement patterns were consistent. However, the movement of the robotic horse tended to be more consistent than real horse movement (Table 3).

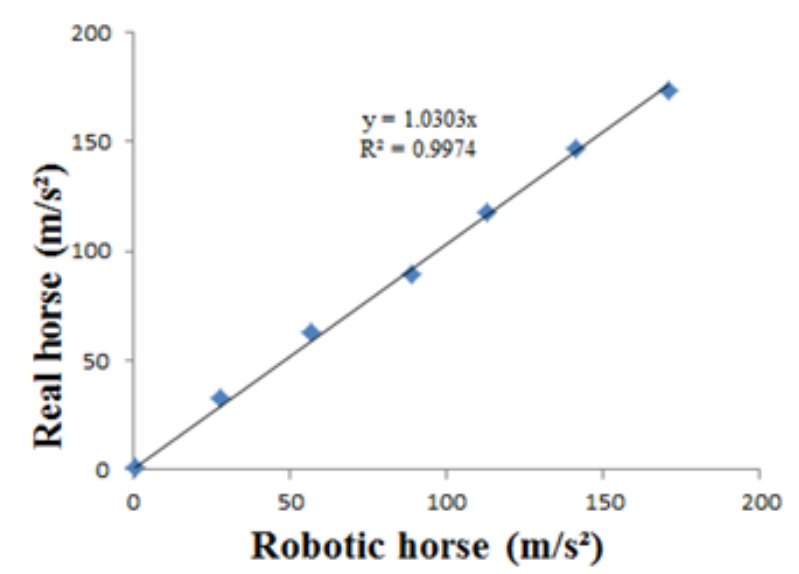

Fig. 2. TPA analysis.

Table 2

Correlational statistics data between robotic and seven real horses' acceleration movements

\begin{tabular}{|c|c|c|c|c|c|c|c|c|}
\hline & & SKIPPY & MONAMI & ROSCO & EQ & LAN & BUG & $\mathrm{K}$ \\
\hline \multirow{3}{*}{ SKIPPY } & Pearson & 1 & -.014 & $-.322^{* *}$ & $.134^{*}$ & $-.205^{* *}$ & $.509^{* *}$ & $-.160^{*}$ \\
\hline & $p$ & & .823 & .000 & .033 & .001 & .000 & .011 \\
\hline & $\mathrm{N}$ & 251 & 251 & 251 & 251 & 251 & 251 & 251 \\
\hline \multirow{3}{*}{ MONAMI } & Pearson & -.014 & 1 & -.064 & -.023 & $.142^{*}$ & .045 & .078 \\
\hline & $p$ & .823 & & .313 & .716 & .024 & .481 & .217 \\
\hline & $\mathrm{N}$ & 251 & 251 & 251 & 251 & 251 & 251 & 251 \\
\hline \multirow{3}{*}{ ROSCO } & Pearson & $-.322^{* *}$ & -.064 & 1 & $-.140^{*}$ & .034 & $-.665^{* *}$ & $.153^{*}$ \\
\hline & $P$ & .000 & .313 & & .027 & .597 & .000 & .015 \\
\hline & $\mathrm{N}$ & 251 & 251 & 251 & 251 & 251 & 251 & 251 \\
\hline \multirow{3}{*}{ EQ } & Pearson & $.134^{*}$ & -.023 & $-.140^{*}$ & 1 & -.039 & $.192^{* *}$ & $-.156^{*}$ \\
\hline & $P$ & .033 & .716 & .027 & & .539 & .002 & .013 \\
\hline & $\mathrm{N}$ & 251 & 251 & 251 & 251 & 251 & 251 & 251 \\
\hline \multirow{3}{*}{ LAN } & Pearson & $-.205^{* *}$ & $.142^{*}$ & .034 & -.039 & 1 & $-.158^{*}$ & .002 \\
\hline & $P$ & .001 & .024 & .597 & .539 & & .012 & .975 \\
\hline & $\mathrm{N}$ & 251 & 251 & 251 & 251 & 251 & 251 & 251 \\
\hline \multirow{3}{*}{ BUG } & Pearson & $.509^{* *}$ & .045 & $-.665^{* *}$ & $.192^{* *}$ & $-.158^{*}$ & 1 & -.122 \\
\hline & $P$ & .000 & .481 & .000 & .002 & .012 & & .053 \\
\hline & $\mathrm{N}$ & 251 & 251 & 251 & 251 & 251 & 251 & 251 \\
\hline \multirow{3}{*}{ K } & Pearson & $-.160^{*}$ & .078 & $.153^{*}$ & $-.156^{7}$ & .002 & -.122 & 1 \\
\hline & $p$ & .011 & .217 & .015 & .013 & .975 & .053 & \\
\hline & $\mathrm{N}$ & 251 & 251 & 251 & 251 & 251 & 251 & 251 \\
\hline
\end{tabular}

*Correlation is significant at the 0.05 level (two-tailed)

**Adjusted correlation is significant at the 0.0001 level (two-tailed) to account for the Type II error 
Table 3

Test-retest reliability of the robotic and real horses

\begin{tabular}{llllllllll}
\hline Reliability/ & Robotic & \multicolumn{2}{l}{ Real Horses } \\
\cline { 2 - 10 } Horse Types & Horse & Monami & Bug & EQ & Lan & K & Skippy & Rosco & \multirow{2}{*}{ Mean } \\
\hline$R^{2}$ & 0.976 & 0.921 & 0.792 & 0.887 & 0.890 & 0.905 & 0.832 & 0.829 & 0.865 \\
\hline
\end{tabular}

\section{Discussion}

The present study provides the first empirical results comparing different robotic and real horse movement patterns using 3-axis accelerometer sensors and demonstrates relatively higher test-retest reliability $\left(R^{2}=0.976\right)$ and excellent validity $\left(R^{2}=0.997\right)$ in the TPA of the resultant acceleration, but poor validity $\left(R^{2}=0.177\right)$ when comparing the $\mathrm{X}$-acceleration movement data between the robotic and real horses. Our novel findings suggest that both robotic and real horse movement patterns are repeatable and valid in the time factor, yet the movement patterns between the robotic and real horses are dissimilar. Such dissimilarity may result from various movement speed and pattern parameters and modifications (e.g., 100 levels) that are built into the robotic horse system [13]. Although a therapist can modify the settings to produce limited random or variable practice within a movement pattern from one mode to other modes in the robotic hippotherapy, the real horse movement is inherently variable, while there is gross similarity between strides and variations among a set of strides as indicated in the correlation data.

Most importantly, these variable features are invaluable for clinicians in designing hippotherapy interventions to enhance the postural and locomotor control required for functional daily activities in children with CP. In a recent robotic study, motor learning was most effective when random or variable perturbing external forces were introduced during locomotor task training [16]. In another study, training with a noise perturbation approach improved tracking performance compared to unassisted training or error-amplification training [17]. Conversely, a randomized clinical trial with incomplete spinal cord injury patients demonstrated that robotic-assisted gait training focused on a passive guidance concept showed minimal improvement in gait velocity following 12 weeks of locomotor training. A possible reason why the robotic passive guidance strategy showed marginal clinical benefits is that correctly-guided kinematic gait feedback provided during the training may deprive the motor learning opportunity, which is acquired by the trial and error paradigm during the cognitive motor learning stage [18].

Intensive repetitive, rhythmic movement is a hallmark of horse movement, like human locomotion. An average horse cadence is reported to be 100 steps per minute at a preferred or medium walking speed, which can serve as a therapeutic challenge for postural stability, since the client needs to adaptively re-stabilize his or her perturbed equilibrium repeatedly [19]. If each hippotherapy session lasts 45 minutes, as many as 3000-5000 repetitions of postural control challenge are presented, which surpasses the number of repetitions that are typically offered in conventional protocols for neurorehabilitation [20]. In this context, the work of Lang and her colleagues might suggest that innovative robotic movement therapy, such as robotic hippotherapy, can amplify the number of repetitions and increase motivation to accomplish those repetitions. Our recent preliminary study showed that robotic hippotherapy could provide rhythmic 2-dimensional postural control and lumbopelvic core stabilization exercise with a large number of repetitions (3000-5000 per each session) [13]. Certainly, this dosage far exceeds both conventional neurorehabilitation (occupational 
therapy/physical therapy) and the minimum requirements for neuroplasticity and motor recovery to occur.

Undoubtedly, the legged robotic horse system with a 6-dgree of freedom (DOF), which mimics a real horse movement as described in details is useful for training novice 'healthy' riders or non-brain injury populations $[12,13]$. However, a greater acceleration movement associated with multiple DOFs in the legged robotic horse or a real horse may pose a great challenge for children with moderate or severe CP. In a real hippotherapy situation, 2 sidewalkers including a therapist usually work together for a safe intervention of children with mild CP. In fact, the present study found that the mean resultant accelerations for a real horse and robotic horse were $3.22 \mathrm{~m} / \mathrm{s}^{2}$ and $0.67 \mathrm{~m} / \mathrm{s}^{2}$, respectively, accounting for approximately 5 times greater acceleration in the real horse than the robotic one. In addition, the option to start low and adjust the movement of the robotic horse below that which is afforded by even the most mildly moving real horse may allow a therapist to accommodate patients with very limited postural control, and then build up the challenge in fine increments as they progress in their strength and coordination.

\section{Conclusion}

The present study demonstrates that the robotic hippotherapy system can provide consistently variable movement patterns that vary from hippotherapy using real horses. Nevertheless, further development and research are warranted to validate the system's safety and therapeutic efficacy in neurological populations with postural instability.

\section{Acknowledgement}

We would like to thank Sandy Rafferty's staff members and horses who have graciously provided their support for data collection at Therapeutic Horsemanship, in Wentzville, MO.

\section{References}

[1] M. Zadnikar and A. Kastrin, Effects of hippotherapy and therapeutic horseback riding on postural control or balance in children with cerebral palsy: A meta-analysis, Developmental Medicine and Child Neurology 53 (2011), 684-691.

[2] D. Silkwood-Sherer and H. Warmbier, Effects of hippotherapy on postural stability, in persons with multiple sclerosis: A pilot study, Journal of Neurologic Physical Therapy 31 (2007), 77-84.

[3] J.L. Encheff, C. Armstrong, M. Masterson, C. Fox and P. Gribble, Hippotherapy effects on trunk, pelvic, and hip motion during ambulation in children with neurological impairments, Pediatric Physical Therapy 24 (2012), 242-250.

[4] J.A. Sterba, B.T. Rogers, A.P. France and D.A. Vokes, Horseback riding in children with cerebral palsy: Effect on gross motor function, Developmental Medicine and Child Neurology 44 (2002), 301-308.

[5] J.Y. Kwon, H.J. Chang, J.Y. Lee, Y. Ha, P.K. Lee and Y.H. Kim, Effects of hippotherapy on gait parameters in children with bilateral spastic cerebral palsy, Archives of Physical Medicine and Rehabilitation 92 (2011), 774-779.

[6] R.L. Casady and D.S. Nichols-Larsen, The effect of hippotherapy on ten children with cerebral palsy, Pediatric Physical Therapy 16 (2004), 165-172.

[7] T.L. Shurtleff and J.R. Engsberg, Changes in trunk and head stability in children with cerebral palsy after hippotherapy: A pilot study, Physical and Occupational Therapy in Pediatrics 30 (2010), 150-163.

[8] T.L. Shurtleff, J.W. Standeven and J.R. Engsberg, Changes in dynamic trunk/head stability and functional reach after hippotherapy, Archives of Physical Medicine and Rehabilitation 90 (2009), 1185-1195.

[9] F. Beinotti, N. Correia, G. Christofoletti and G. Borges, Use of hippotherapy in gait training for hemiparetic post-stroke, Arq. Neuropsiquiatr. 68 (2010), 908-913. 
[10] C.W. Lee, S.G. Kim and M.S. Yong, Effects of hippotherapy on recovery of gait and balance ability in patients with stroke, Journal of Physical Therapy Science 26 (2014), 309-311.

[11] D.R. Lee, N.G. Lee, H.J. Cha, Y.S. O, S.H. You, J.H. Oh and H.S. Bang, The effect of robo-horseback riding therapy on spinal alignment and associated muscle size in MRI for a child with neuromuscular scoliosis: An experimenter-blind study, Neurorehabilitation 29 (2011), 23-27.

[12] G. Carbone and M. Ceccarelli, Legged Robotic Systems, in: Cutting Edge Robotics, ARS Scientific Book, Wien, 2005, pp. 553-576.

[13] R. Eskola and H. Handroos, Novel horseback riding simulator based on 6-DOF motion measurement, a motion base, and interactive control of gaits, Advanced Robotics 26 (2013), 1249-1257

[14] G. Zurek, K. Dudek, I. Pirogowicz, A. Dziuba and M. Pokorski, Influence of mechanical hippotherapy on skin temperature responses in lower limb in children with cerebral palsy, Journal of Physiology and Pharmacology 59 (2008), 819-824.

[15] S.M. Michaelsen, R.P. Gomes, A.P. Marques, L.C. Rodrigues, N.G. Borges Junior, R. Claudino and M.J. dos Santos, Using an accelerometer for analyzing a reach-to-grasp movement after stroke, Motriz. Journal of Physical Education 19(2013), 746-752

[16] L. Marchal-Crespo, J. Schneider, L. Jaeger and R. Riener, Learning a locomotor task: with or without errors? Journal of Neuro Engineering and Rehabilitation 11 (2014), 17-21.

[17] J. Lee and S. Choi, Effects of haptic guidance and disturbance on motor learning: Potential advantage of haptic disturbance, Haptics Symposium, 2010, 335-342.

[18] E.C. Field-Fote and K.E. Roach, Influence of a locomotor training approach on walking speed and distance in people with chronic spinal cord injury: A randomized clinical trial, Physical Therapy 91 (2011), 48-60.

[19] H.M. Clayton, Walk this way: Learn to discern the fine points of this all important basic gait, Veterinary Connection, 2002, 39-42.

[20] C.E. Lang, J.R. MacDonald and C. Gnip, Counting repetitions: An observational study of outpatient therapy for people with hemiparesis post-stroke, Journal of Neurologic Physical Therapy 31 (2007), 3-10. 